\title{
Optimization of a chemooptical surface plasmon resonance based sensor
}

Jos van Gent, Paul V. Lambeck, Harrie J. M. Kreuwel, Gerrit J. Gerritsma, Ernst J. R. Sudhölter, David N. Reinhoudt, and Theo J. A. Popma

\begin{abstract}
A surface plasmon based chemooptical sensor has been optimized by the use of computer simulation programs. Calculated and experimentally measured performances are in good agreement, showing the value of the simulation tool.
\end{abstract}

\section{Introduction}

In modulation type chemooptical (CO) sensors the chemical domain is connected with the optical domain by a CO transducing layer, in which a specific chemical concentration is transduced into a change of the dielectric function of this layer, via a reversible binding of the chemical species to the interface. By applying appropriate optical measuring principles, this change in dielectric function is transformed into a change in energy of a light beam.

As was first pointed out by Liedberg et al., ${ }^{1}$ surface plasmon resonance (SPR) based optical measuring principles is very interesting, because it shows good possibilities for the realization of fast sensors with high sensitivity. Fast response can be achieved by using a very thin $\mathrm{CO}$ transducing layer and high sensitivity by the use of surface plasmons, in which the light energy is highly concentrated in the transducing layer. Kreuwel et al. ${ }^{2}$ developed an SPR-based solid state microsensor by coupling the surface plasmon supporting structure to a planar waveguiding system, thus abandoning the rather bulky optical equipment that is commonly used.

For practical uses the required sensor performance has to be specified in terms of selectivity, response time, measurement range, and sensitivity. The selectivity and the response time are controlled by the properties of the transducing layer, the measurement range and sensitivity by both the optical measuring system and the transducing layer.

In this paper we shall concentrate on the aspect of sensitivity, as far as it is controlled by the optical system. For the most simple surface plasmon sup-

All authors are with University of Twente, P.O. Box 217, NL-7500 AE Enschede, The Netherlands. E. J. R. Sudhölter and D. N. Reinhoudt are in the Laboratory of Organic Chemistry; J. van Gent, P. V. Lambeck, H. J. M. Kreuwel, G. J. Gerritsma, and T. J. A. Popma are in the Laboratory of Materials Science.

Received 15 May 1989.

0003-6935/90/192843-07\$02.00/0.

(C) 1990 Optical Society of America. porting structure the sensitivity can be estimated from analytical functions as described in Sec. II. However for more complex optical structures, it has to be calculated numerically.

In this paper we will show that the results of numerical simulations can be used both to optimize the layer structure and to obtain more insight into the behavior of the sensor. Starting with the most simple surface plasmon supporting structure, a CO sensor system is built up by adding subsequent layers stepwise. For each intermediate structure the simulation is compared with the experimental performance. Finally a CO sensor for vapor with basic properties is described.

\section{Surface Plasmon Resonance}

A surface plasmon is an electromagnetic wave propagating along the interface of two materials, one of them having a negative dielectric constant (e.g., silver or gold). ${ }^{3}$ The electric field $\mathbf{E}$ of the most simple surface plasmon supporting structure, i.e., one consisting of two semi-infinite layers (Fig. 1), can be described analytically. The electrical field of a plasmon propagating along the interface $x=0$ in the $z$-direction is given by

$$
\mathbf{E}(x, z, t)=\mathbf{E}^{0}(x) \text { expi }\left(\omega t-\hbar_{z} z\right),
$$

where $\omega=$ angular frequency, and $\tilde{k}_{z}=k_{z}^{\prime}+i k_{z}^{\prime \prime}$, the propagation constant.

The field amplitude $\mathbf{E}^{0}(x)$ reaches a maximum at the interface and decays exponentially in the adjacent half spaces (Fig. 1). The decay constants are in the range of $10^{-1}-10^{-3} \mathrm{~nm}^{-1}$, indicating the large field concentration in the transducing layer. The propagation constant of a surface plasmon propagating along the interface of two semi-infinite layers depends on the dielectric properties of the constituting layers and is given by the dispersion relation ${ }^{3}$ :

$$
\tilde{k}_{z}=\frac{\omega}{c} \sqrt{\frac{\tilde{\epsilon}_{\mathrm{Ag}} \tilde{\epsilon}_{D}}{\tilde{\epsilon}_{\mathrm{Ag}}+\tilde{\epsilon}_{D}}},
$$

where $c=$ velocity of light, and $\tilde{\epsilon}_{\mathrm{Ag}}, \tilde{\epsilon}_{D}=$ the complex 


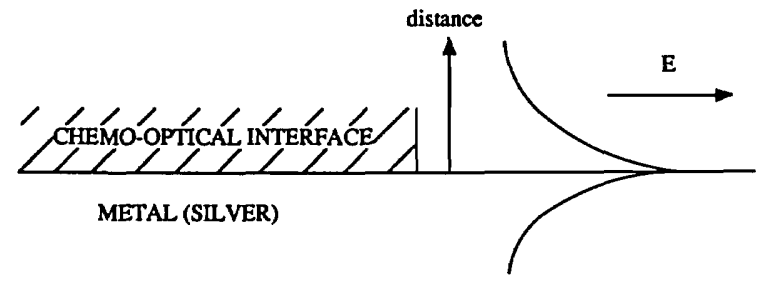

dielectric constants of the silver and the dielectric layer, respectively.

In the $\mathrm{CO}$ sensor the surface plasmon supporting structure consists of at least four layers: a substrate, a silver film, the $\mathrm{CO}$ transducing layer, and the environment. In Sec. $V$ we will discuss the addition of a fifth layer, having a high dielectric constant, intermediate between the silver layer and the transducing layer. For these multilayer structures $\mathbf{E}_{x}^{0}$ and $k_{z}$ have to be calculated numerically (see Sec. III). Essential is the change in $k_{z}$, caused by the change of $\epsilon_{t}$ (the dielectric constant of the $\mathrm{CO}$ transducing layer) originating from reversible binding of the chemical entity in the transducing layer, that is measured in surface plasmon based $\mathrm{CO}$ sensors. The large field confinement along the silver transducing layer interface permits the use of relatively thin interface layers, resulting in a fast sensor response.

The commonly used experimental setup, the Kretschmann configuration, ${ }^{4}$ is shown symbolically in Fig. 2. The surface plasmon supported by the silver sensing layer interface will only be excited from the incident laser light beam if the condition

$$
k_{z}=n_{p} k_{0} \sin (\theta)=N_{\text {eff }} k_{0}
$$

is obeyed, where

$$
k_{0}=\frac{2 \pi}{\lambda_{0}}
$$

and $n_{p}=$ refractive index of the prism

$\lambda_{0}=$ laser beam wavelength in vacuum $(\mathrm{m})$

$\theta=$ angle of incidence (see Fig. 2).

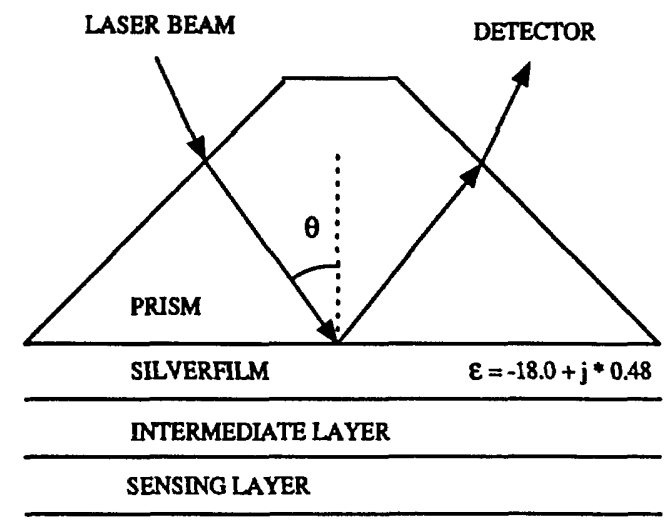

EXTERNAL ENVIRONMENT

Fig. 2. Experimental setup for the detection of surface plasmon resonance curves (Kretschmann configuration).

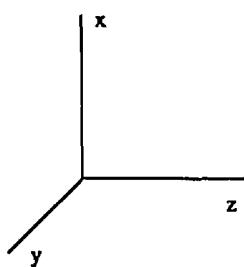

Fig. 1. Electric field profile of a surface plasmon.
The surface plasmon resonance is experimentally observed as a sharp minimum of the light reflectance on variation of $N_{\text {eff }}=n_{p} \sin (\theta)$ (see Fig. 3).

As is common in resonance phenomena the resonance is spread by damping. Damping results in both an increased width and a decreased height of the resonance peak, and decreases the accuracy of the measured angle of minimum reflection. The damping originates from the imaginary parts of the dielectric constants of the silver and the transducing layers, the scattering originating from the surface roughness and the coupling of the surface plasmon with the radiation field in the prism. In terms of sensitivity the possibility of utilizing a surface plasmon supporting optical structure for measuring the $\epsilon$-change of the transducing layer, depends on the magnitude of the shift $\Delta k_{z}^{\prime}$ caused by a given change $\Delta \epsilon(\lambda)$ of the $C O$ transducing layer and on the damping proportional to the imaginary part of the propagation constant $k_{z}^{\prime \prime}$. This can be expressed by a relative figure of merit $S$ defined as

$$
S=\frac{\Delta k_{z}^{\prime}}{k_{z}^{\prime \prime}} \text {. }
$$

The values of $\Delta k_{z}^{\prime}$ and $k_{z}^{\prime \prime}$ can be obtained from the $R(\theta)$ reflectance curves (Fig. 3 ), because they are proportional to the shift of the resonance peak (presented as a dip in the reflection curve), and its halfwidth, respectively ${ }^{6}$ When we assume that a change in intensity of only $1 \%$ of the intensity of the incident beam can be recorded by our measuring equipment, a more practical figure of merit would be:

$$
S=\frac{\Delta k_{z}^{\prime}}{w_{1 \%}}
$$

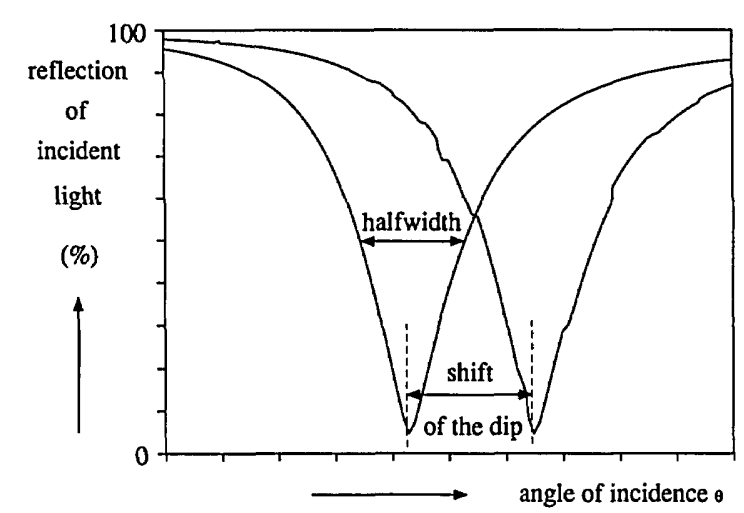

Fig. 3. How to find the relative figure of merit $S$ from the $\mathrm{R}(\theta)$ reflectance curves. 
where $w_{1 \%}$ is the width of the surface plasmon dip at the intensity level $1 \%$ above the minimum intensity.

The plasmon structure should be designed to maximize $S$, i.e., a large shift of the SPR dip and a sharp and deep minimum in the reflectance curve. Computer simulation programs have been developed to perform this optimization.

\section{Computer simulation programs}

Simulation programs can be very helpful to predict the behavior of surface plasmon sensors. In addition these simulations give more insight into the physical background of these devices.

The PASCAL computer program SP-DIP was developed for the theoretical calculation of surface plasmon dips. This program, based on Abeles method, ${ }^{5}$ calculates the reflectance of a multilayer plasmon supporting structure (maximum ten layers) as a function of the angle of incidence $\theta$ (see Fig. 2), presenting this dependence as $R-N_{\text {eff }}$ plots $\left(N_{\text {eff }}=n_{\text {prism }} \sin \theta\right)$. The interfaces between the layers are assumed to be parallel and completely smooth and the layers to be homogeneous. The prism can be treated as a semi-infinite layer. The noninfinite layers can have any positive thickness.

The program has wider uses. Not only surface plasmon modes, but also guided modes of the layer structure can easily be found. In this way it is possible to determine modes in multilayer structures, containing highly dispersive materials.

To calculate the field distributions in the multilayer structure in the resonant case, which appear to be very helpful to understand the influence of the parameters on the sensor performance, another simulation program (FIELD ${ }^{6}$ ) has been developed.

Input data for both programs consist of the complex dielectric constants for all layers and the thicknesses for the nonsemi-infinite layers. Sample runs of both programs are presented in the next sections.

\section{Prism-Silver Layer-Transducing Layer Structure}

As a starting point the simplest SPR supporting layer structure, consisting of a prism, a silver layer, and a semi-infinite dielectric layer has been studied. Attention was paid to the surface plasmon that propagates along the silver-dielectric interface. The experiments were restricted to transducing layers with negligible losses at the applied wavelength. The following values of the input parameters have been used:

$\begin{array}{lll} & \text { thickness } & \tilde{\epsilon} \\ \text { prism } & \infty & 1.7996 \\ \text { silver layer } & \text { varied } & -18+i .0 .48 \\ \text { dielectric layer } & \infty & \text { pure real, varied }\end{array}$

$\left(\lambda_{0}=632.8 \mathrm{~nm}\right.$,

He-Ne laser)

The position of the dip has been calculated as a function of the thickness of the silver film (Fig. 4). It can be concluded from these calculations that silver films of more than $40 \mathrm{~nm}$ thickness can be regarded as semi-infinite. In that case the propagation constant of the plasmon can be calculated from Eq. (2).

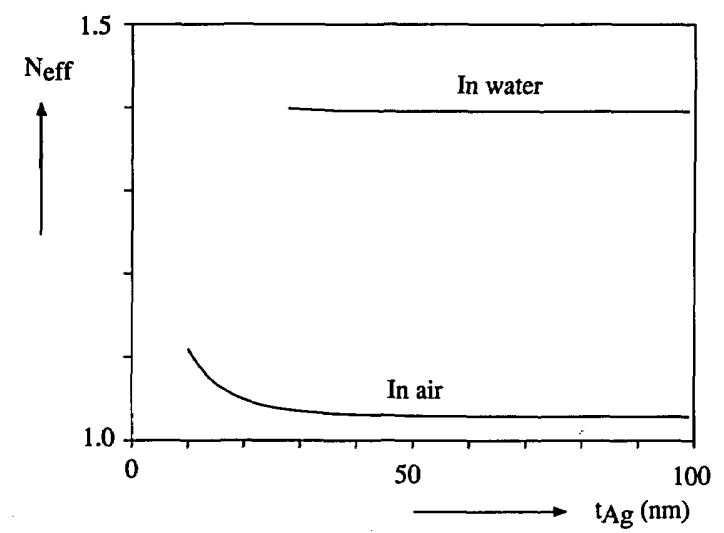

Fig. 4. Position of the dip, expressed in terms of $N_{\text {eff }}$, as a function of the thickness of the silver film $\left(t_{\mathrm{Ag}}\right)$ for the systems prism-silverair and prism-silver-water.

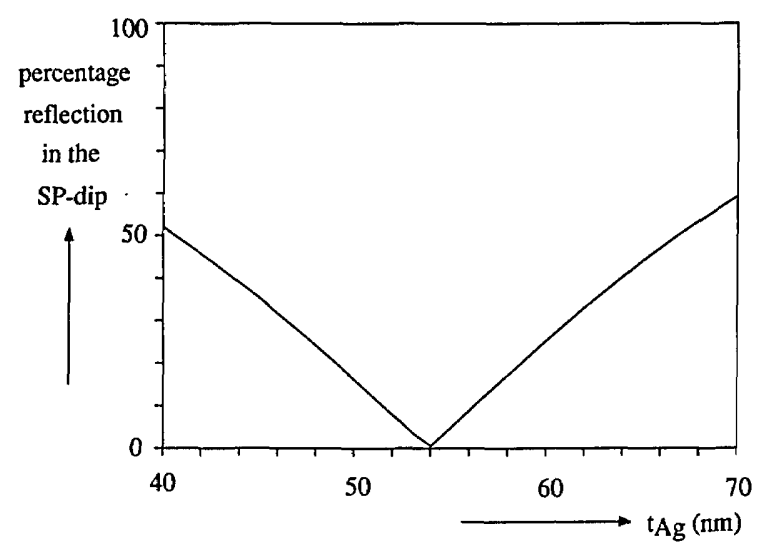

Fig. 5. Depth of the dip as a function of the thickness of the silver layer for the system prism-silver-air.

Using the computer program SP-DIP the thickness of the silver film $t_{\mathrm{Ag}}$ was optimized as to the deepest minimum in the reflection of the dip. The halfwidth and the position of the SPR dip appear to vary only little with the thickness of the silver film. The optimal thickness of the silver film was thus calculated to be 54 nm (see Fig. 5) for the system silver-air. For this thickness losses caused by the coupling between the plasmon and the radiation field in the prism (increasing with decreasing thickness) and losses caused by dissipation (decreasing with decreasing thickness) and scattering are equal. ${ }^{6}$ Since our model does not account for scattering losses the calculated optimal thickness is somewhat too large. However, the introduced error is small. The optimal thickness is rather critical. In other systems this optimum appears to differ slightly, but the differences are negligible in the systems we have used. Therefore in all further calculations and experiments we have maintained $t_{\mathrm{Ag}}=54$ nm.

To test the correctness of the results of the computer program we measured the surface plasmon resonance curve of the system consisting of a silver film evaporated by an e-beam on a glass substrate. This substrate 
was placed on top of the prism (SF-10 glass, $n=1.72$ ) with diiodomethane $\left(\mathrm{CH}_{2} \mathrm{I}_{2}, n=1.68\right)$ as a matching liquid between prism and substrate, thus forming one optical unit. The $N_{\text {eff }}$ of the dip as a function of the dielectric constant of a semi-infinite layer of air, water, or acetone in contact with the silver surface (Fig. 6) can be described nicely by the theory. In this case Eq. (2) gives the same results as the computer program. With respect to the system prism-silver-air the difference between the calculated and the measured $N_{\text {eff }}$ values (1.0293 and 1.0282 respectively, corresponding with an angle difference of $\sim 0.02^{\circ}$ ) is of the order of the experimental error. The experimentally measured dip halfwidth (see Fig. 7), however, exceeds the calculated one with a factor of 1.2. This difference may be attributed to scattering losses, caused by the roughness of the glass surface, because these losses are not taken into account in our model. In the case of water or acetone the experimental dips are much wider than calculated. The reason for this widening is not yet known. Further experiments were carried out on slide glass substrates, unless otherwise indicated.

\section{Intermedlate Layer}

The surface plasmon sensor which will be discussed is based on the commonly used structure given in Fig. 2. There were a number of reasons to add an intermediate $\mathrm{ZrO}_{2}$ layer between the silver film and the chemooptical transducing layer, viz.

1. Protection of the silver film.

The silver surface is very reactive and especially sulphur containing compounds in the environment cause a rapid degradation of the silver film. Provided that the protecting film shows no pinholes, even a very thin film (10 $\mathrm{nm}$ or less) of organic or inorganic materials is shown to be able to protect the silver film against these influences, at least for our measuring period of 6 months.

2. Immobilization of the transducing layer.

The oxidic intermediate layer is very appropriate for chemically binding of the organic transducing layer.

3 . Concentration of the electric field.

By virtue of its high dielectric constant (4.2 in the case of $\mathrm{ZrO}_{2}$ ) the presence of the intermediate layer will result in a greater confinement of the electric field in the interface region. It might be expected that field enhancement in the neighboring transducing layer would lead to a greater sensitivity. However, in Sec. VI it will be shown that this is not correct.

To compare the SP-resonance curves for the system of Fig. 2, calculated from the simulation program, with the experimental ones we prepared substrate-silver$\mathrm{ZrO}_{2}$ specimens $\left(t_{\mathrm{Ag}}=54 \mathrm{~nm}, t_{\mathrm{ZrO}_{2}}=0,10,20,30 \mathrm{~nm}\right)$. In a vacuum system containing both a silver source and $\mathrm{a} \mathrm{ZrO}_{2}$ source, the silver and the $\mathrm{ZrO}_{2}$ layer were evaporated by an e-beam in one run, thus preventing contamination of the silver layer.

Figure 8 shows a good agreement in the positions of the calculated and measured reflection minima. Also in the case of these systems, the experimentally obtained halfwidth of the SP-resonance curves are great-

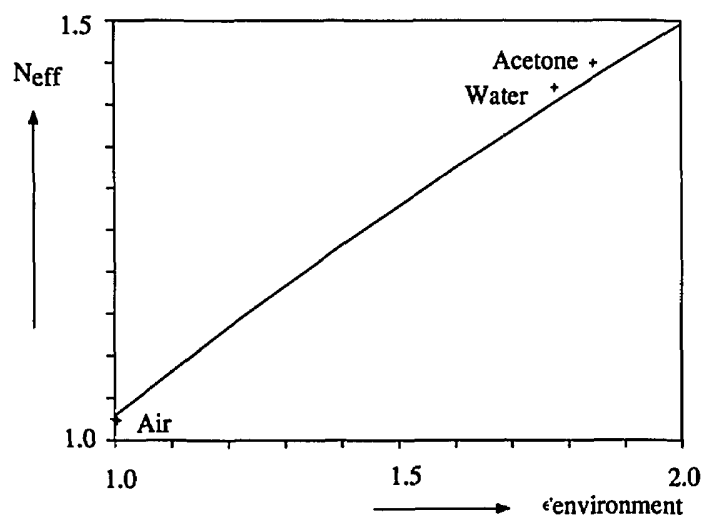

Fig. 6. Calculated (line) and measured (+) position of the surface plasmon dip in the system prism-silver-dielectric, where the dielectric is air, water or acetone.

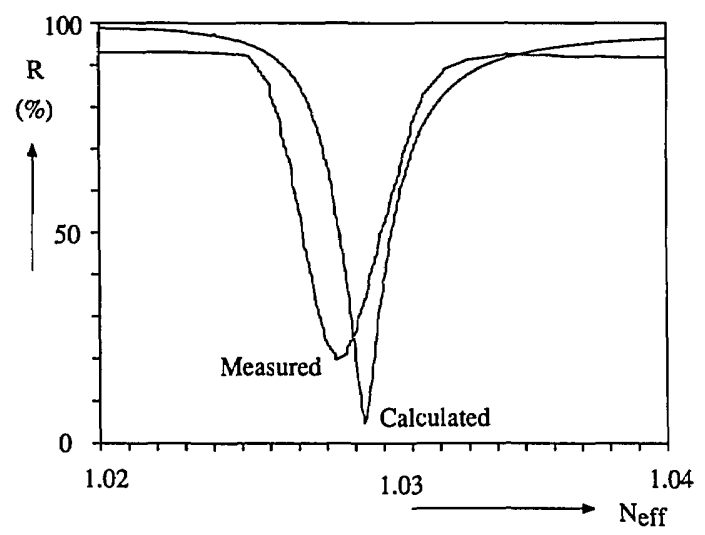

Fig. 7. Calculated and measured surface plasmon dispersion curves for the system prism-silver-air (slide glass substrates).

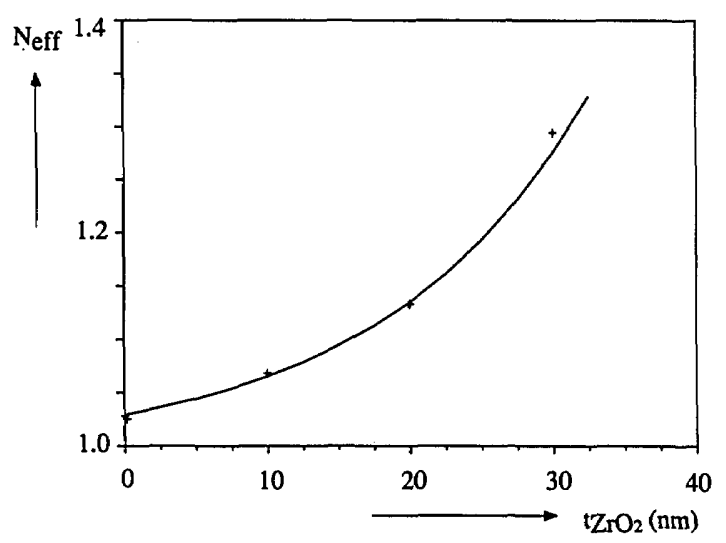

Fig. 8. Calculated (line) and measured $(+)$ positions of the surface plasmon dip of the system prism-silver- $\mathrm{ZrO}_{2}$-air.

er than the calculated ones (Fig. 9). The calculated field intensity distributions of these structures are depicted in Fig. 10, clearly showing the expected increase of the electrical field in the interface region of $\mathrm{ZrO}_{2}$ and air. 


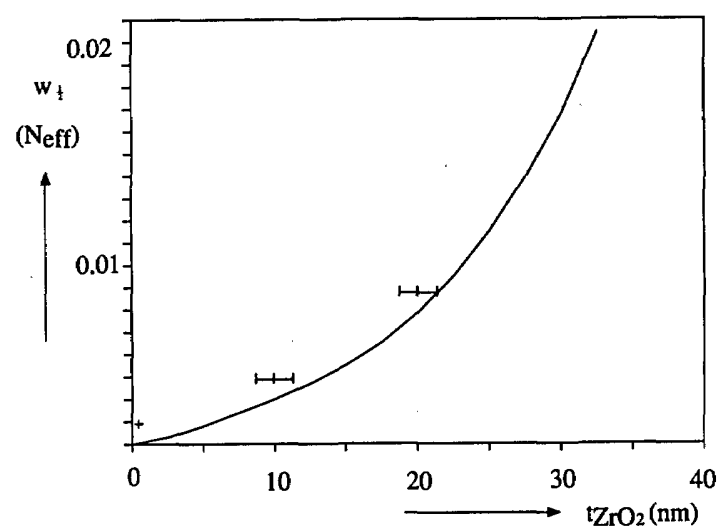

Fig. 9. Halfwidth of the surface plasmon dip as a function of $t_{\mathrm{ZrO}_{2}}$ in the system prism-silver- $\mathrm{ZrO}_{2}$-air.

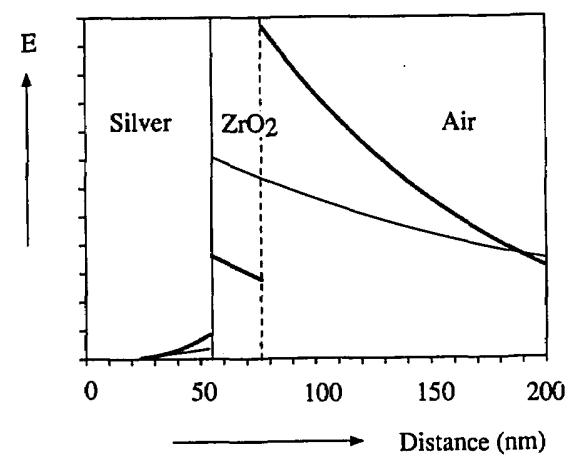

Fig. 10. Electric field distribution of a surface plasmon in the systems prism-silver-air (-) and prism-silver- $\mathrm{ZrO}_{2}$-air (-).

\section{CO Transducing Layer}

To compare the simulated and experimentally obtained SP resonance curves of a complete sensor system, we had to produce a real transducing layer. In our systems a bromo cresol purple (BCP) layer (BCP is a well-known $\mathrm{pH}$-indicator), prepared by careful vacuum evaporation, performs the transduction function. Simulation of the complete sensing system requires knowledge of the real part $\epsilon^{\prime}$ and the imaginary part $\epsilon^{\prime \prime}$ of the dielectric function (or because $\tilde{\epsilon}=\tilde{n}^{2}$ equivalently the real part $n^{\prime}$ and the imaginary part $n^{\prime \prime}$ of the refractive index) and of the thickness of the BCP-film. The $\epsilon^{\prime}$ and $\epsilon^{\prime \prime}$-values can be obtained from the experimentally recorded absorption spectra [Fig. 11(a)], using the relation ${ }^{7}$

$n^{\prime \prime}(\lambda)=c_{1} \lambda \epsilon_{\lambda}(\lambda)$

where $n^{\prime \prime}=$ the imaginary part of the refractive index.

$c_{1}=a$ constant $\left(\mathrm{mol} \mathrm{cm} \mathrm{dm} \mathrm{m}^{-3} \mathrm{~nm}^{-1}\right)$,

$\lambda=$ the wavelength ( $\mathrm{nm})$, and

$\epsilon_{\lambda}=$ the wavelength dependent molar extinction coefficient of the dye $\left(\mathrm{dm}^{3} \mathrm{~mol}^{-1}\right.$ $\mathrm{cm}^{-1}$ )

The variation of $n^{\prime}$ with $\lambda$ can be calculated from $n^{\prime \prime}(\lambda)$ using the Kramers-Kronig relations. ${ }^{7}$ For this pur-
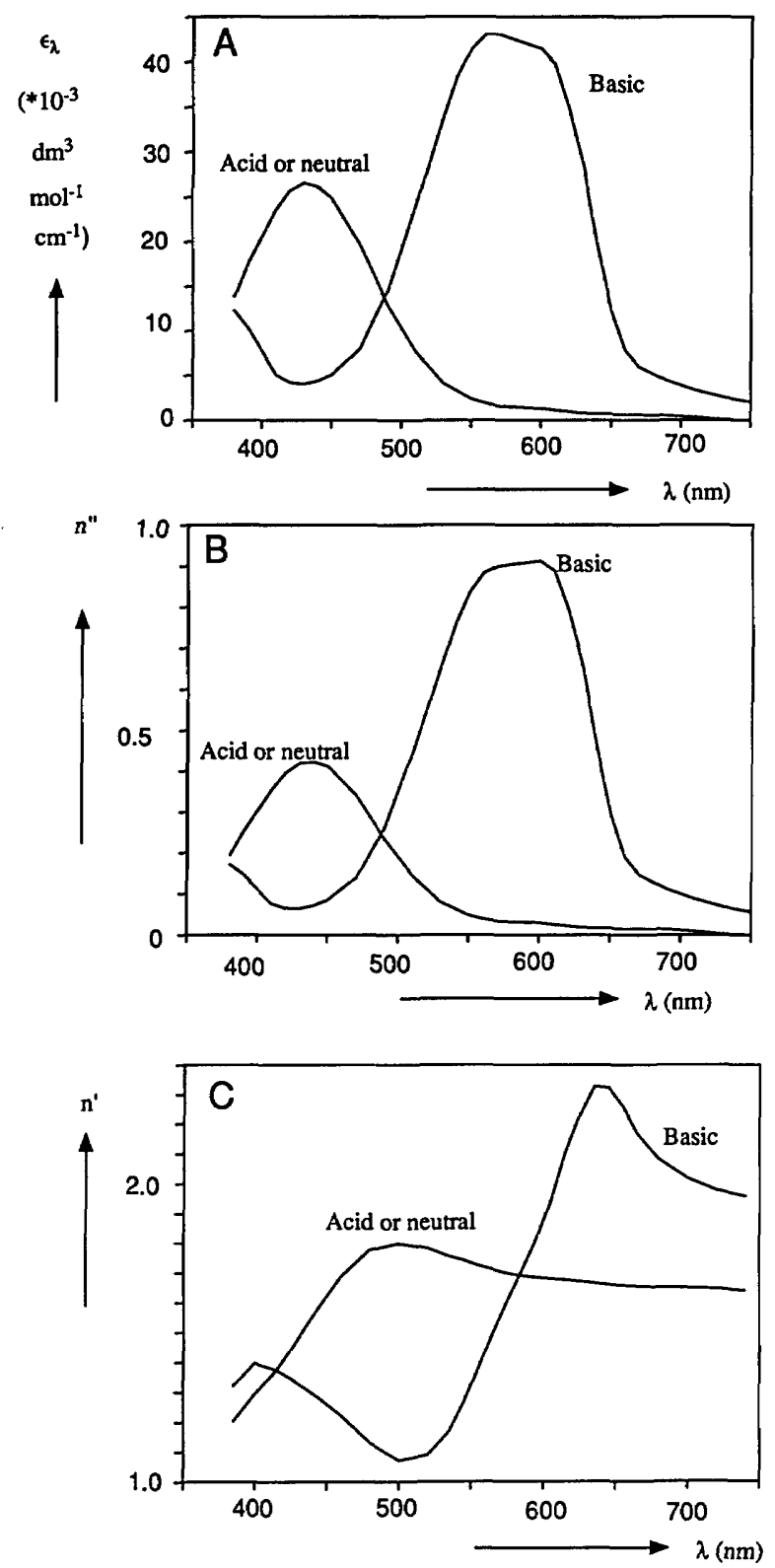

Fig. 11. A. Absorption spectra of the acid (or neutral) and basic form of BCP. B. Imaginary part of the refractive index $\left(n^{\prime \prime}\right)$ as a function of the wavelength for the acid and basic forms of BCP. C. Calculated real part of the refractive index $\left(n^{\prime}\right)$ as a function of the wavelength for the acid and basic forms of BCP.

pose the PASCAL computer program REFRAC was developed, based on the Kramers-Kronig relations, Akima Point Interpolation, ${ }^{8}$ and Delves Principal Value Integration. ${ }^{9}$ The absolute level of $n^{\prime}(\lambda)$ can only be obtained by supplying $n^{\prime}\left(\lambda_{1}\right)$ in the input file, where $\lambda_{1}$ is a value for $\lambda$. This value, determined by ellipsometry, was $1.76 \pm 0.02$ at $632.8 \mathrm{~nm}$ for the acid form of BCP. Figure 11(b) shows the imaginary part and Fig. 11(c) the real part of the refractive index of BCP as calculated by REFRAC. Because of relationship (7) the $n^{\prime \prime}(\lambda)$-curve [Fig. 11(b)] and the absorption spectrum [Fig. 11(a)] look very similar. Near an absorption 
maximum $n^{\prime}(\lambda)$ is almost proportional to the first derivative of $n^{\prime \prime}(\lambda)$.

To obtain a large sensitivity a wavelength has to be chosen for which the change of $n^{\prime}$ on change from the acidic to the basic form is large, while $n^{\prime \prime}$ in both forms is small. It can be deduced from Figs. 11(b) and 11(c) that for this dye the wavelength of a $\mathrm{He}-\mathrm{Ne}$ laser $(632.8 \mathrm{~nm})$ is well suited.

A basic environment for the experiments was created with ammonia vapor. When the atmosphere was made basic a color change could be visually observed. Returning to the acid/neutral form was achieved by aeration.

For the comparison of the simulated and experimentally obtained SPR dispersion curves multilayer structures were prepared, consisting of:

(1) Substrate, consisting of slide glass.

(2) Silver film, $54 \mathrm{~nm}$ thick.

(3) Intermediate layer, consisting of $\mathrm{ZrO}_{2}$, thickness 0-10-20-30 nm.

(4) Transducing layer (BCP), $4.6 \mathrm{~nm}$ thick.

(5) External environment, consisting of air or a mixture of air and ammonia vapor.

Calculated and experimentally obtained shifts of the reflection minima, upon a change from the acid to the basic form, and the values of the halfwidth are depicted in Figs. 12 and 13 respectively.

The shift of the position of the dip when the BCP layer is exposed to ammonia vapor was in rather good agreement with the expected shift (Fig. 12). The experiment could be reproducibly repeated several times. The sensor has a very fast response time (only a few seconds), which means that this configuration behaves as a sensor for ammonia (and other basic gases) in air.

The measured dips of these systems were much wider than the calculated ones (Fig. 13). Therefore, we believe that the structure of the sensing layer is not homogeneous, the average thickness of the layer is 4.6 $\mathrm{nm}$, but on microscale relatively large variations are possible.

Although all measured halfwidths are larger than the calculated ones, the calculated and measured differences between the halfwidth of the reflection curves of the neutral and basic BCP layers show a better correspondence (Fig. 14). This is not surprising, because the halfwidth originates from a summation of scattering losses, losses in the silver layer, and losses in the transducing layer, the first two of these being independent of the transducing layer state. Thus the halfwidth difference between both forms will be determined by the difference in transducing layer losses in both states, which the simulation model (contrary to the scattering) accounts for very well.

In Fig. 15 the calculated figure of merit $S$ is presented as a function of the thickness of the $\mathrm{ZrO}_{2}$ layer. Contrary to the prediction given in Sec. V, S does not increase with increasing $\mathrm{ZrO}_{2}$ layer thickness at all. Reconsideration of the field distributions presented in Fig. 10 reveals, that by applying a $\mathrm{ZrO}_{2}$ intermediate

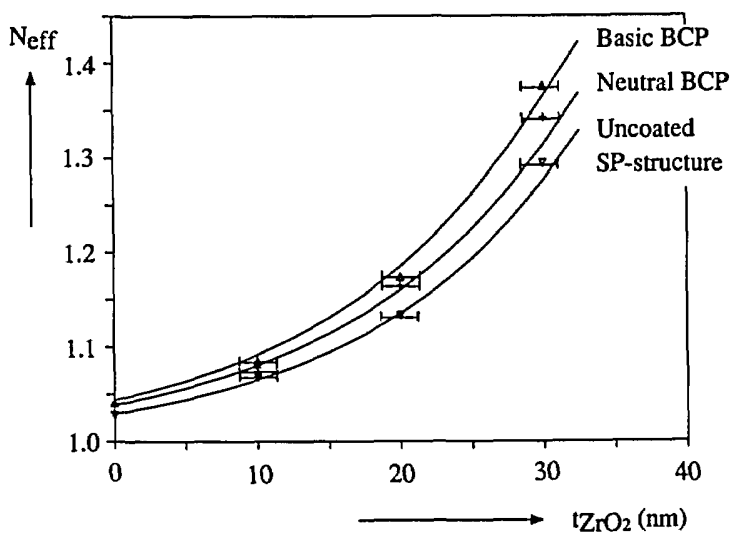

Fig. 12. Calculated (lines) and measured (symbols) positions of the surface plasmon dip as a function of the thickness of the $\mathrm{ZrO}_{2}$ layer for the system prism-silver $(54 \mathrm{~nm})-\mathrm{ZrO}_{2}$ (variable)-BCP $(4.6 \mathrm{~nm})-$ air (+:without BCP, $\triangle: B C P$ in neutral environment, $\nabla: B C P$ in basic environment).

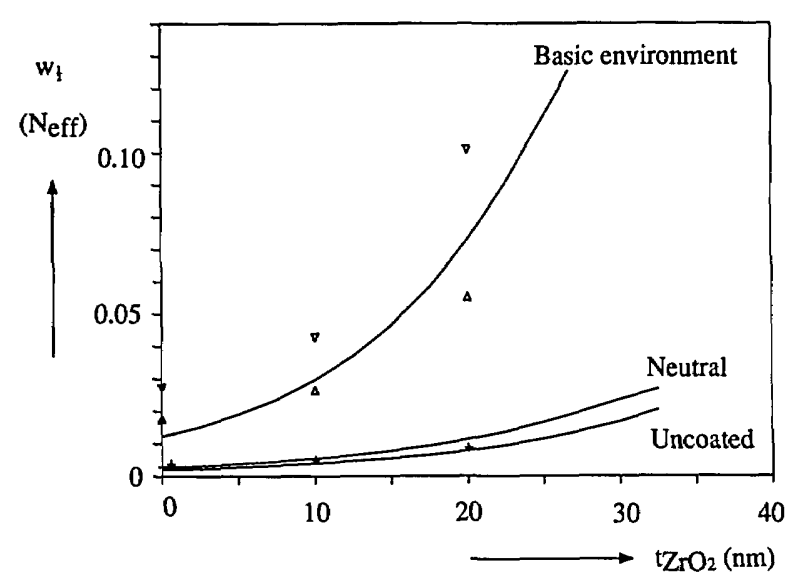

Fig. 13. Calculated (lines) and measured (symbols) halfwidths of the surface plasmon dip as a function of the thickness of the $\mathrm{ZrO}_{2}$ layer for the system prism-silver $(54 \mathrm{~nm})-\mathrm{ZrO}_{2}$ (variable)-BCP (4.6 $\mathrm{nm}$ )-air ( $+:$ without BCP, $\triangle: B C P$ in neutral environment, $\nabla: B C P$ in basic environment.

layer the electric field energy in the silver layer increases as well, resulting in an enlarged damping.

It has to be concluded from simulation results that the negative effect of the increased damping on the figure of merit $S$ at least cancels the positive effect of the field enhancement in the transducing layer. The difference between calculated and measured $S$ values must be attributed to additional dip broadening caused by scattering, which in turn is caused by surface roughness. Considering also the protective function of the $\mathrm{ZrO}_{2}$ layer, a thickness of $\sim 20 \mathrm{~nm}$ appears to be optimal.

Because the presence of the intermediate layer causes a decrease of the field energy in the environment, it will be helpful to decrease the influence of a change in dielectric function of the environment on the SP resonance curve.

In Sec. IV it was concluded that the optimal thick- 


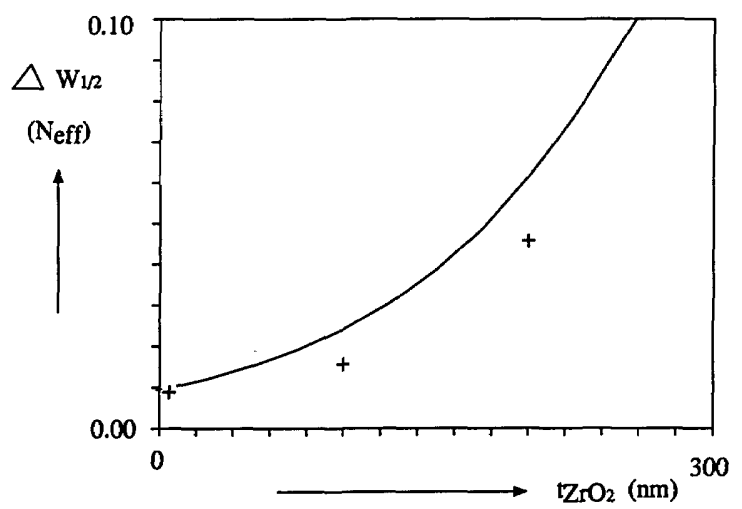

Fig. 14. Change in halfwidth of the reflection dips of the BCPsensor system resulting from the change from acid to basic environment.

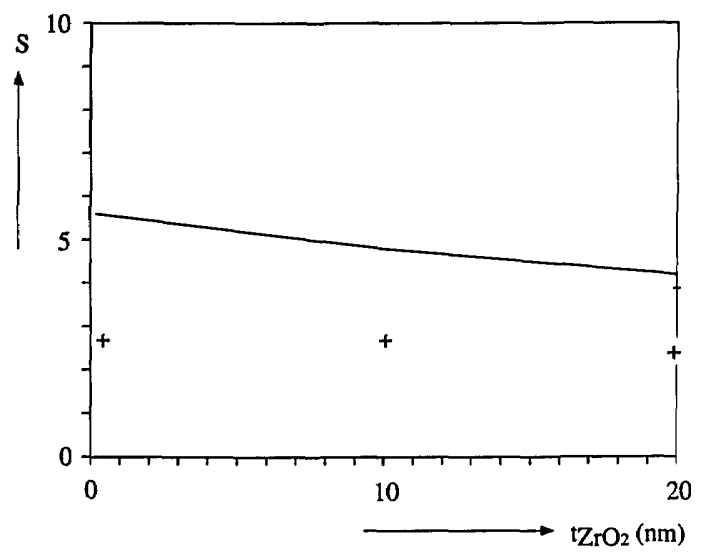

Fig. 15. Calculated (line) and measured ( + ) relative figure of merit $S$ as a function of the intermediate $\mathrm{ZrO}_{2}$ layer.

ness of the silver layer in a prism-silver-air system is $54 \mathrm{~nm}$. We have learned from simulations that the optimal silver layer thickness for the complete sensor system is in good approximation not influenced by the intermediate or the sensing layer.

The maximum shift in the effective refractive index for the sensor system presented here is 0.033 , corresponding to a difference in the angle of incidence $\theta$ of $\sim 1.6^{\circ}$. Assuming a resolution of $0.02^{\circ}$, this implies a maximal resolution of $1.3 \%$ of full scale.

\section{Summary and Conclusions}

Computer assisted simulation is a powerful tool in optimizing a multilayer $\mathrm{CO}$ surface plasmon resonance sensor as to the aspect of sensitivity.

Good correspondence is obtained between calculated and experimental shifts of the resonance peaks. To achieve a good fit in resonance peak width, scattering, originating from surface roughness, has to be incorporated in the model. Addition of a thin $\mathrm{ZrO}_{2}$ layer, intermediate between the silver layer and the $\mathrm{CO}$ transducing layer, not only offers the necessary protection of the silver layer, but also reduces the interference of changes in refractive index of the environment. An SPR supporting structure is realized, provided with a thin BCP layer, thus being sensitive to ammonia vapor. This structure offers good prospects as ammonia sensitive solid state sensor, showing a response time in the order of seconds.

Further research on the quantification of the $\mathrm{NH}_{3}$ sensitivity as well as the development of other chemical sensitive layers for various types of $\mathrm{CO}$ microsensors, especially ion-sensors based on chromoionophores ${ }^{10}$ is in progress.

We thank B. Hurenkamp for preparing the glass substrate substrates and prisms, $\mathrm{H}$. Krabbe and C. A. $M$. Harteveld for evaporating the silver and $\mathrm{ZrO}_{2}$ layers, and R. Verhoeven for writing the computer program REFRAC.

Jos van Gent also works in the Laboratory of Organic Chemistry.

\section{References}

1. C. Nylander, B. Liedberg, and T. Lind, "Gas Detection by Means of Surface Plasmon Resonance," Sensors and Actuators 3, 79-88 (1982).

2. H. J. M. Kreuwel, P. V. Lambeck, J. van Gent, and Th.J.A. Popma, "Surface Plasmon Dispersion and Luminescence Quenching applied to Planar Waveguide Sensors for the Measurement of Chemical Concentrations," Proc. Soc. Photo-Opt. Instrum. Eng. 798, 218-224 (1987).

3. E. Burstein, A. Hartstein, J. Schoenwald, A. A. Maradudin, D. L. Mills, and R. F. Wallis, "Surface Polaritons-Electromagnetic Waves at Interfaces," in Proceedings of the First Taormina Research Conference on the Structure of Matter, E. Burstein and F. de Martini, eds. (Pergamon 1974), pp. 89-108.

4. E. Kretschmann, "The Determination of the Optical Constants of Metals by Excitation of Surface Plasmons," Z. Phys. 241, 313324 (1971), in German.

5. R. Ullrich, "Theory of the Prism-Film Coupler by Plane-Wave Analysis," J. Opt. Soc. Am. 60, 1337-1350 (1970).

6. H. J. M. Kreuwel, "Planar Waveguide Sensors for the Chemical Domain," Ph.D. Thesis, U of Twente, Enschede, The Netherlands, pp. 52 and further (1988).

7. R. Loudon, The Quantum Theory of Light (Clarendon, Oxford, 1981) pp. 64 and further.

8. H. Akima, "A New Method of Interpolation and Smooth Curve Fitting based on Local Procedures," Journal of the Assoc. Comput. Mach. 17, 589-602 (1970).

9. L. M. Delves, "The Numerical Evaluation of Principal Value Integrals," Comput. J. 10, 389-391 (1967).

10. J. van Gent, E. J. R. Sudhölter, P. V. Lambeck, G. J. Gerritsma, Th.J.A. Popma, and D. N. Reinhoudt, "A Chromogenic Crown Ether as a Sensing Molecule in Optical Sensors for the Detection of Hard Metal Ions," J. Chem. Soc., Chem. Commun., 893-895 (1988). 